\title{
Étude iconographique des monnaies autonomes frappées par Colophon-sur-Mer : nouveaux acquis
}

\section{Nuran Şahin}

\section{(2) OpenEdition \\ 1 Journals}

\section{Édition électronique}

URL : http://journals.openedition.org/anatoliaantiqua/379

DOI : $10.4000 /$ anatoliaantiqua.379

\section{Éditeur}

IFEA

Édition imprimée

Date de publication : 1 mai 2016

Pagination : 147-162

ISBN : 9782362450648

ISSN : 1018-1946

\section{Référence électronique}

Nuran Şahin, «Étude iconographique des monnaies autonomes frappées par Colophon-sur-Mer:

nouveaux acquis », Anatolia Antiqua [En ligne], XXIV | 2016, mis en ligne le 11 décembre 2018, consulté le 16 février 2021. URL : http://journals.openedition.org/anatoliaantiqua/379; DOI : https://doi.org/ 10.4000/anatoliaantiqua.379 


\section{ANATOLIA ANTIQUA ESKI ANADOLU}

\section{XXIV}

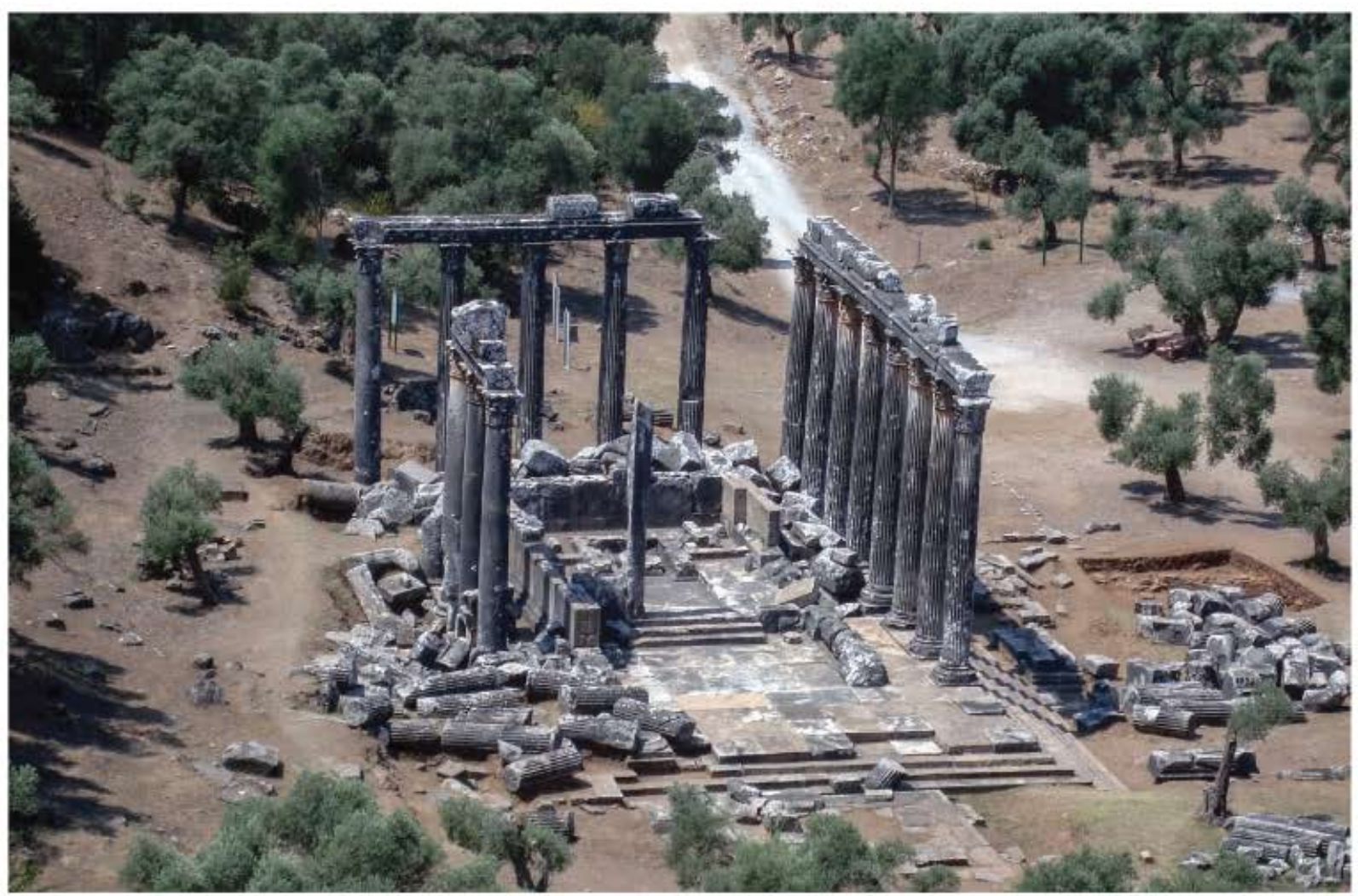

INSTITUT FRANÇAIS D'ETUDES ANATOLIENNES GEORGES-DUMEZIL

CNRS USR 3131

DE BOCCARD 


\section{TABLE DES MATIERES}

Ergul KODAS,

Le surmodelage du crâne au Néolithique au Proche-Orient : techniques de surmodelage et

expérimentations

Isabella CANEVA et Eric JEAN,

Mersin-Yumuktepe : une mise au point sur les derniers travaux

Turan EFE et Bérengère PERELLO,

Second Millenium site distribution and pottery of Inland Northwestern Anatolia

Antoine PEREZ,

Amida 6 : Antiochos IV, le "Hanigalbat" et la Sophène

Ergün LAFLI et Hadrien BRU,

Inscriptions et monuments funéraires gréco-romains d'Anatolie occidentale

Oğuz TEKİN et Aliye EROL-ÖZDİZBAY,

Coins from Allianoi excavations: Campaign of 2001

Nuran ŞAHIN,

Etude iconographique des monnaies autonomes frappées par Colophon-sur-Mer :

nouveaux acquis

Vera SAUER,

Konventionelle Individualität. Zur Münzprägung nordanatolischer Städte in der römischen

Kaiserzeit

Oğuz TEKIN,

Balance weights in the collection of the Anatolian Civilizations Museum in Ankara

\section{CHRONIQUES DES TRAVAUX ARCHEOLOGIQUES EN TURQUIE 2015}

Çiğdem MANER,

Preliminary report on the third season of the Konya-Ereğli Survey (KEYAR) 2015

Dominique BEYER, Isabelle CHALIER et Françoise KIRNER,

Rapport préliminaire sur les travaux de la mission archéologique de Zeyve Höyük-Porsuk 2015 ...

Sami PATACI et Ergün LAFLI,

Field surveys in Ardahan in 2015

Jean-Charles MORETTI avec la collaboration de Nicolas BRESCH, Isabel BONORA,

Jean-Jacques MALMARY et Olivier RISS,

Claros, le temple d'Apollon : travaux réalisés en 2015

Serdar Hakan ÖZTANER,

La basilique civile de Nysa du Méandre

Abuzer KIZIL, Koray KONUK, Patrice BRUN, Laurent CAPDETREY, Raymond DESCAT,

Pierre FROHLICH, Didier LAROCHE, Enora LE QUERE, Francis PROST, Baptiste VERGNAUD,

Eurômos : rapport préliminaire sur les travaux réalisés en 2015

O. HENRY et E. ANDERSON, Chr. BOST, Ö. ÇAKMAKLI, F. CEDERLING, A. COMMITO,

M. CORMIER-HUGUET, A. COUTELAS, A. DOLEA, D. ERGENÇ, A. FRECCERO,

A. FREJMAN, P. LEBOUTEILLER, F. LESGUER, D. LÖWENBORG, V. LUNGU,

Fr. MARCHAND-BEAULIEU, A. SITZ, P. DE STAEBLER, B. VERGNAUD, 


\section{ETUDE ICONOGRAPHIQUE DES MONNAIES AUTONOMES FRAPPEES PAR COLOPHON-SUR-MER : NOUVEAUX ACQUIS}

Cet article a pour but de fournir une étude iconographique des monnaies autonomes frappées par Colophon-sur-Mer découvertes à Claros plus particulièrement lors des fouilles menées de 2001 à $2015^{1}$.

Parmi les monnaies, le plus important groupe porte une représentation d'Homère. Une monnaie a été trouvée pendant les fouilles dirigées par Louis Robert $^{2}$, dix autres ont été découvertes entre 1988 et 1997 pendant les fouilles dirigées par Juliette de La Genière $^{3}$. Les fouilles menées entre 2001 et 2013 ont fourni vingt-deux monnaies de ce type ${ }^{4}$.

Au droit est représenté Homère trônant à gauche, un rouleau dans la main gauche, le menton appuyé sur la main droite, avec deux noms de magistrats soit $\mathrm{A} \Pi \mathrm{O} \Lambda \Lambda \mathrm{A} \Sigma^{5}$, soit $\Pi \mathrm{r}^{\gamma} \mathrm{EOO} \Sigma^{6}$ dans le champ à gauche. Au revers on trouve un personnage debout à droite, une patère dans la main droite, un instrument à cordes dans la gauche. La présence d'Homère sur les monnaies de Colophon n'est pas étonnante car d'après Strabon ${ }^{7}$, Homère était originaire de Colophon. Ces monnaies sont très importantes pour l'histoire du sanctuaire de Claros, Observons tout d'abord, la datation des monnaies de Colophonsur-Mer portant une représentation d'Homère. Leur datation pose des problèmes. Le premier travail paru sur la datation de ce groupe a été effectué par B.V. Head qui a situé ce groupe vers 300 av. J.-C. ${ }^{8}$. Alors que J.G. Milne a proposé une datation entre 190 et $130^{\circ}$. Par la suite ce groupe a été situé entre le $2^{\mathrm{e}} \mathrm{s}$. et le $1^{\text {er }}$ s. av. J.-C. dans la $S N G$ Lewis ${ }^{10}$. Plus tard, $\mathrm{Ph}$. Kinns ${ }^{11}$ a proposé une datation plus précise à 50 av. J.-C. Cette dernière datation a été acceptée par Z. Çizmeli Öğün ${ }^{12}$ qui souligne que le deuxième réaménagement du système monétaire de Colophon commence à partir de la fin du $2^{\mathrm{e}}$ siècle av. J.-C., parallèlement aux activités architecturales dans le sanctuaire $^{13}$. Il faut également indiquer que dix monnaies étudiées par Z. Çizmeli Öğün provenant des sondages $1 \mathrm{~B}, 4 \mathrm{C}, 4 \mathrm{BC}, 3 \mathrm{~F}, 3 \mathrm{~L}$ ont été trouvées dans le sol 4. Les données de ce sol peuvent donc fournir une information importante pour la datation de ces monnaies. Un nombre assez important des trouvailles provenant de ce sol sont les bols à reliefs (dits mégariens). La chronologie de ce sol est très importante pour l'organisation de l'aire des sacrifices et du dispositif pour les hécatombes. Parce qu'il s'agit d'un remblai apporté lors de l'installation des blocs à anneaux, les céramiques les plus récentes qu'il contient (les bols à reliefs), donnent un terminus post quem pour les travaux ${ }^{14}$. J. de La Genière souligne que les activités de Polemaios et de Ménippos

*) nuran.sahin@ege.edu.tr.; nurans2005@msn.com

1) Je remercie chaleureusement J.-Ch. Moretti qui a bien voulu relire le texte de cet article et nous faire profiter ainsi de ses précieux conseils.

2) Une monnaie provenant de l'autel hellénistique d'Apollon a été découverte en 1951 lors des fouilles menées par L. Robert.

3) Çizmeli Öğün $2007: 221, n^{\text {os }} 41-42$.

4) Delrieux $2014: 145-146, \mathrm{n}^{\text {os }} 144-150$; trois monnaies découvertes en 2013 (inédites).

5) Sept monnaies découvertes entre 2003 et 2011, contenaient le nom de AПO $\Lambda$ A $\Sigma$. Voir Delrieux $2014: 145-146, \mathrm{n}^{\mathrm{os}} 144-150$.

6) Trois monnaies découvertes en 2006 et 2007, portaient le nom de Пr $\Theta E O \Sigma$. Voir Önol 2009 : Fig. VII, n ${ }^{\text {os }} 117$ - 119; Delrieux $2014: 146, n^{\text {os }} 150-153$; c'est aussi le cas d'une monnaie non publiée provenant du sondage MN12 et trouvée en 2012. Sur les noms de magistrats attestés sur les monnaies de Colophon voir Kinns 1980 : 336.

7) Strabon XIV, 1, 28.

8) BMC Ionia : 41- 42, Fig. VIII. 10.

9) Milne 1941 : Période VII, groupe E 178-9 ; SNG Cop. Ionia : 184-185.

10) SNG Lewis : 892-893 ; Sear $1979: 400,4357$.

11) Kinns 1980: AE 24, $\mathrm{n}^{\text {os }} 176-177$.

12) Cizmeli Öğün 2007: 221, ns 41-42.

13) Çizmeli Öğün $2011: 8 ; 2012: 63$.

14) Delattre, La Genière et Şahin $2003: 18$ 
(deux évergètes) $)^{15}$ réalisées dans le troisième tiers $\mathrm{du} 2^{\mathrm{e}} \mathrm{s}$. av. J.-C. ${ }^{16}$ pourraient donner un terminus ante quem pour la céramique hellénistique, plus particulièrement pour les bols à reliefs (mégariens) trouvés dans la strate de préparation du sol foulé lors des hécatombes. Elle précise que les bols à reliefs devraient être antérieurs à la fin du $2^{\mathrm{e}} \mathrm{s}$. av. J.-C. donc au réaménagement du sanctuaire ${ }^{17}$. La stratigraphie et la chronologie du sol montrent clairement que la datation de 50 av. J.-C. avancée par Z. Çizmeli Öğün pour les dix monnaies provenant de cette strate n'est pas acceptable.

İ. Önol ${ }^{18}$ et finalement F. Delrieux ${ }^{19}$ ont aussi accepté la datation de Ph. Kinns. Cependant cinq monnaies, étudiées dernièrement $\left(\mathrm{n}^{\mathrm{o}} 157-161\right)^{20}$ par F. Delrieux, provenant des sondages NO13, RS $14 / 15^{21}$ et O13 ont été trouvées aussi avec une céramique hellénistique abondante notamment des bols à relief, toujours dans la strate de préparation du sol. Il faut indiquer également que trois monnaies $\left(\mathrm{n}^{\circ} 157-159\right)$ provenant du sondage NO13 ont été découvertes avec quatre monnaies (Kinns période II = Milne période III, groupe A 128-141) ${ }^{22}$ datées entre 320 et 294 av. J.-C. sous des tuiles moulées. Ce groupe illustre l'activité de l'atelier de Colophon l'Ancienne avant son arrêt ${ }^{23}$.

A la lumière des arguments avancés ci-dessus, il faut évidemment s'interroger sur la datation de ce groupe donnée par $\mathrm{Ph}$. Kinns qui l'a daté du point de vue stylistique et d'après les monnaies de Smyrne. Sur les monnaies en bronze de Smyrne dite Homè- reion ${ }^{24}$ dont les plus anciennes sont datées du début $\mathrm{du} 2^{\mathrm{e}} \mathrm{s}$. av. J.-C. Apollon est toujours représenté au droit et Homère au revers ${ }^{25}$ alors qu'Homère est toujours représenté au droit des monnaies en bronze de Colophon-sur-Mer. Rappelons aussi que le gymnase de Colophon-sur-Mer s'appelait aussi Homèreion ${ }^{26}$ au moment où le décret de Colophon-sur-Mer pour Athènaios fut adopté ${ }^{27}$. Les adolescents et jeunes gens s'exerçaient dans l'Homèrion au moins à l'époque hellénistique ${ }^{28}$. D'après Platon "Homère était l'éducateur de la Grèce". Les écoliers apprenaient par cœur de larges extraits de son œuvre, qui a été considérée comme essentielle pour la formation des jeunes $^{29}$. Homère de Colophon patronnait aussi en quelque sorte la formation de la jeunesse à Colophon-sur-Mer. ${ }^{30}$ La statue d'Homère provenant de Claros, datée du deuxième quart du $2^{\mathrm{e}} \mathrm{s}$. av. J.-C. ${ }^{31}$, l'illustre. La question qui se pose est de savoir quand et pourquoi Homère est devenu pour ainsi dire le dieu de l'éducation de Notieis ? Peut-on supposer que cela a relevé de l'idéologie du royaume des Attalides, parce que la dynastie des Attalides attachait une grande importance aux gymnases aussi bien à Pergame que dans les autres villes du royaume? Les Attalides ont versé beaucoup d'argent pour les gymnases. Les gymnases ont été considérés comme des centres de formation de la jeune génération par les rois de Pergame. L'objectif était de faire assimiler aux jeunes l'idéologie du royaume ${ }^{32}$. Rappelons aussi qu'il existait aussi une fondation attalide assurant des banquets pour ceux (néoi et presbyteroi)

15) Pour Polemaios et Menippos, voir Robert et Robert $1989: 103-104$.

16) Pour la datation des deux décrets, voir Robert et Robert 1989: 9.

17) La Genière $2003: 193, \mathrm{Pl}$. XI, 3, fig. $28, \mathrm{n}^{\text {os }} 11-14$.

18) Önol $2009: 55-57, n^{\text {os }} 113-122$.

19) Delrieux $2014: 145$

20) Delrieux $2014: 146, n^{\text {os }} 157-161$

21) Dans le catalogue de Delrieux, la monnaie $n^{\circ} 160$ a été donnée comme le nom de personne indéterminé au revers alors que le nom d'une personne est visible ( $\mathrm{A} \Pi \mathrm{O} \Lambda \Lambda \mathrm{A} \Sigma)$ au droit mais pas au revers. Voir Delrieux $2014: 179, \mathrm{n}^{\circ} 160$. $18-99$

22) Ce groupe a été présenté avec quatre-vingt-un exemplaires dans le catalogue de F. Delrieux. Voir Delrieux 2014: 139-142, nºs

23) Amandry $1992: 95-96$.

24) Strabon, XIV, 1, 37 (C 646).

25) Milne 1941 : 97, 221, 241, 307, 324, 359 ; SNG Ionia : 2167, 2169 ; SNG Mysia-Ionia : 3175-3180 ; Izmir Ticaret Tarihi Müzesi. Antik Dönem Sikke Koleksiyonu, 2011 : nos 10-13, 15-16, 18-25, 27-28, 30-42, 46, 48.

26) Pour l'emplacement de l'Homèreion voir Macridy et Picard $1915: 37-38$, n. 7 ; Demangel et Laumonier 1923 : 354, 359 . L. Robert (1999: 184, n. 41) suppose qu'il y avait un sanctuaire d'Homère dans un gymnase ce qui expliquerait que le gymnase de Colophon-sur-Mer s'appelait Homèreion.

27) La datation de ce décret pose problème parce qu'il a été daté d'abord vers 200 av. J.-C par M. Holleaux (1906:350, col 27). L. Robert (1970: 154, n 1) avait proposé une date un peu avant 197 av. J.-C. Cependant le décret a été daté entre 180-150 av. J.-C. par Chr. Habicht (1969: 28, n. 5). Gauthier (2006 : 473-494) a proposé une date plus proche de celle de Habicht, entre 180 et 160. Pour la datation du décret de Colophon pour le prince Athénaios, voir Debord $2013: 11$.

28) Gauthier $2006: 492$.

29) Xénophon, Le Banquet III, 5.

30) Gauthier $2006: 492$.

31) Robert $1999: 182,184$

32) Malay $1992: 152-153$. 
qui fréquentaient le gymnase après l'éphébie ${ }^{33}$. Dans ces conditions il ne serait pas étonnant que le gymnase de Colophon-sur-Mer ait été nommé $\mathrm{Ho}$ mèreion par les Attalides. On sait également par le décret pour Athènaios qu'il y avait les statues des membres de la famille d'Attale I ${ }^{\text {er }}$, plus particulièrement autour de la statue de la reine Apollonis dans le sanctuaire de Claros $^{34}$. Les travaux réalisés dans le sondage $1 \mathrm{H}$ en 1994 ont mis au jour une série de fondations de monuments honorifiques datées du dernier quart du $3^{\mathrm{e}}$ siècle au début du $2^{\mathrm{e}}$ siècle, détruites lors du début de la construction du dispositif pour les hécatombes ${ }^{35}$. L'alignement de ces monuments honorifiques a montré qu'ils ont été placés du côté d'un passage existant à l'époque hellénistique $^{36}$. Les travaux menés toujours dans le sondage $1 \mathrm{H}$ en 1996 ont mis au jour la suite de ces monuments ainsi qu'une grande exèdre semi-circulaire orientée elle aussi vers le $\mathrm{Sud}^{37}$. On a suggéré que ces monuments avaient été dédiés aux Séleucides ou à la famille des Attalides par les Colophoniens ${ }^{38}$. La présence du décret de Colophon-sur-Mer pour le prince Athénaios, le dernier fils d'Attale I ${ }^{\mathrm{er}}$, permet d'attribuer ces monuments à la famille des Attalides plutôt qu'à celle de Séleucides. A la paix d'Apamée, Colophon-sur-Mer fut reconnue libre et exempte de tribut mais ce ne fut pas le cas de Colophon l'Ancienne, parce que les Colophoniens habitant à Colophon l'Ancienne étaient entrés dans l'alliance avec les Séleucides (le roi Antiochos III) ${ }^{39}$. Les Colophoniens-sur-Mer (Notieis $)^{40}$ avaient de bons rapports avec les Attalides, comme l'atteste notamment le décret en l'honneur d'Athénaios.

Abordons enfin l'iconographie des trente deux monnaies en bronze de Colophon-sur-Mer ${ }^{41}$ provenant de Claros sur lesquelles Homère a été représenté comme type de droit. Le premier travail d'ensemble des monnaies de Colophon a été fait par J.G. Milne ${ }^{42}$. Mais les identifications iconographiques qu'il a faites sont parfois erronées. L'erreur la plus évidente est la cithare présentée comme type de revers sur les monnaies de Colophon l'Ancienne. La cithare a été interprétée comme une lyre par J.G. Milne ${ }^{43}$ alors que l'instrument à corde figuré sur les monnaies est toujours une cithare et jamais une lyre.

Une autre identification faite par J.G. Milne pour les monnaies que nous étudions est à écarter. Il avait identifié le personnage figuré au revers comme Apollon à la lyre ${ }^{44}$. Il faut encore souligner que le personnage tient une cithare dans la main gauche et non une lyre. Il est très intéressant que l'identification de J.G. Milne comme Apollon ait été acceptée par les numismates sans discuter l'iconographie de la figure $^{45}$. Aujourd'hui, les identifications de J.G. Milne nous obligent donc à faire une étude iconographique à la lumière des nouvelles découvertes de Claros.

Deux monnaies en bronze de Colophon-sur-Mer provenant du sondage MN12 ${ }^{\text {os }} 3.2$ et 3.4 (Fig. 1a$\mathrm{b}$ et 2a-b) et aussi une monnaie en bronze provenant du sondage ST 13-14 № 3.176 (Fig. 3a-b) ont été trouvées durant la campagne de 2012. Sur la monnaie de MN12 $\mathrm{n}^{0} .3 .2^{46}$, qui est bien conservée, Homère figure comme type de droit, tourné à gauche, assis sur un trône, le menton appuyé sur la main droite. Il tient un rouleau dans la main gauche. Le nom de personne $\Pi$ r $\Theta E O \Sigma$ apparaît dans le champ à gauche. $\mathrm{Au}$ revers une figure debout tient une cithare dans la main gauche et une patère dans la main droite. L'ethnique KO $\Lambda O \Phi \Omega N I \Omega N$ apparaît dans le champ à gauche. Cette figure mérite une étude plus détaillée. La figure a été représentée de trois-quarts, avec des cheveux coiffés en chignon, et elle porte un long kredemnon $(\kappa \rho \varepsilon ́ \delta \varepsilon \mu \nu o v)^{47}$, qui descend derrière son

33) Robert et Robert $1989: 99-100$.

34) Gauthier $2006: 480$.

35) La Genière $1996: 267$.

36) La Genière $1996: 267$; Şahin $2003: 94$, fig. XXIV, 2.

37) La Genière $1998: 247$, fig. $1, n^{\circ} 12$.

38) La Genière 1998: 247.

39) Polybe XXI, 45, 4. Tite Live, XXXVIII, 39-41.

40) Pour les deux communautés, voir Rousset 2014 : 51-54.

41) D’après Kinns (1980 : 334), ces monnaies de Colophon ont été frappées par Colophon-sur-Mer et non par Colophon l'Ancienne et elles constituaient un nouveau type.

42) Milne 1941

43) Milne 1941: 36-49, groupe période II, groupe A 13-19, groupe B 20-23 groupe C 24-28, groupe D 39-40.

44) Milne 1941 : 79-80, groupe E 178, 179.

45) SNG Ionien, $\mathrm{n}^{\circ} 2017$; SNG Mysien-Ionien, $\mathrm{n}^{\text {os }}$. 2915-2919; Kinns 1980 : 176-177 ; Çizmeli Öğün 2007 : 221, $\mathrm{n}^{\text {os }} 41-42$; Önol 2009: $\mathrm{n}^{\text {os }} 113-122$; Delrieux $2014: 145$, n $^{\text {os }} 144-161$.

46) $Æ 19$ mm. 5.03 g. 3 h.

47) Cette sorte de mantelet, qui couvre la tête, laissait à découvert le visage. Andromaque et Hécube portaient le kredemnon (Homère, Iliade, XXII, 406. 470-473), ainsi que Pénélope (Homère, Odyssée, XVI, 413-416). On le rencontre aussi dans les hymnes homériques (Hymne homérique à Déméter II, 40-42). 

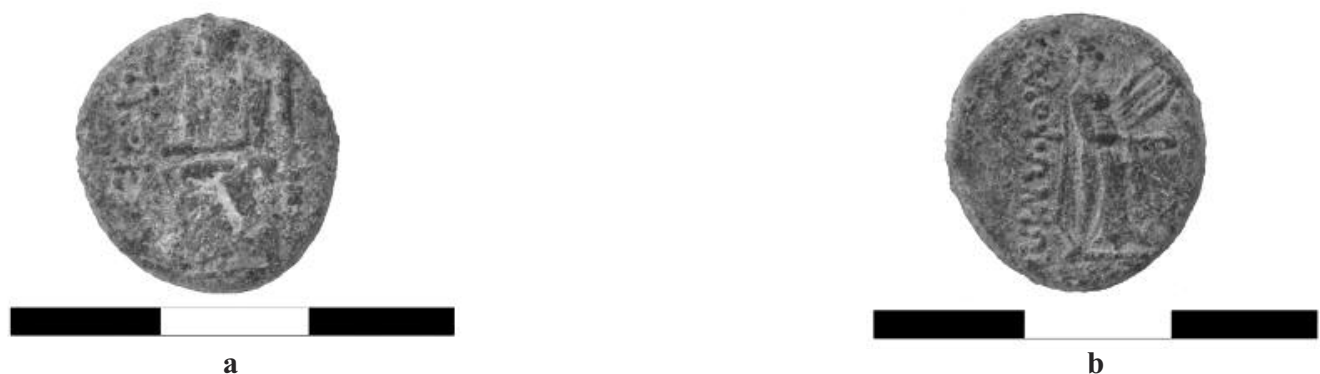

Fig. 1 : Monnaie provenant du sondage MN12. No 3.2 (cliché N. Şahin).
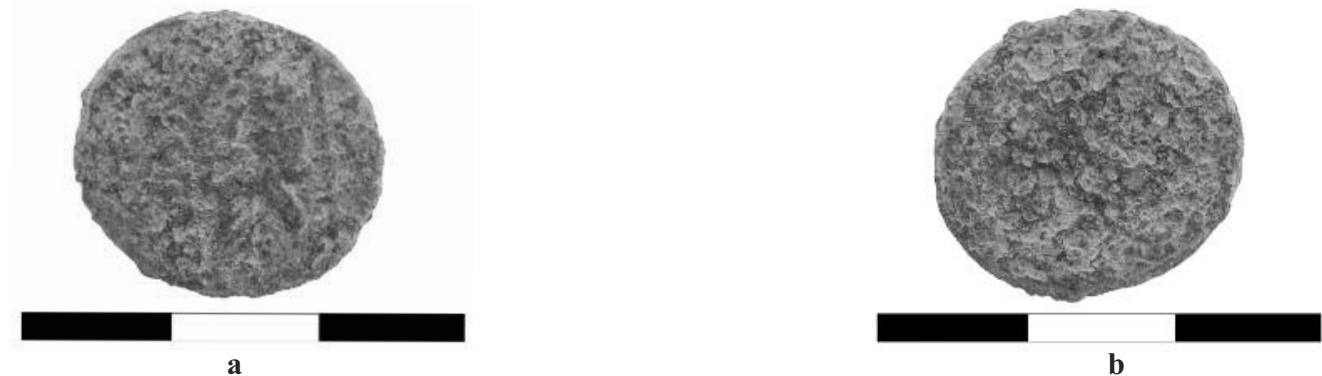

Fig. 2 : Monnaie provenant du sondage MN12. nº 3.4 (cliché N. Şahin).
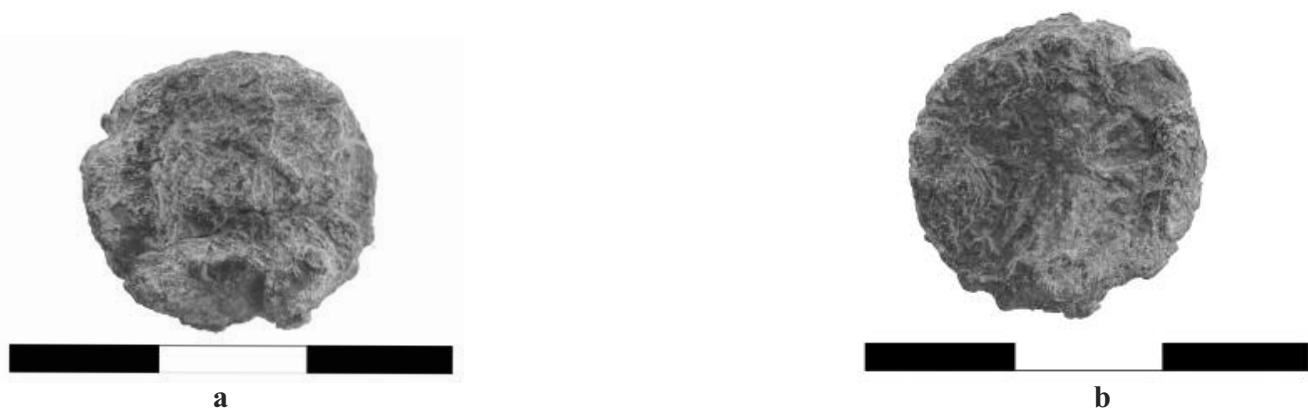

Fig. 3 : Monnaie provenant du sondage ST 13-14 nº 3.176 (cliché N. Şahin).

dos. Il faut noter également que les figurines des courotrophes provenant de Claros sont toujours coiffées du kredemnon ${ }^{48}$. La figurine en terre cuite de Léto provenant de Claros portant les jumeaux dans ses bras est aussi coiffée d'un kredemnon couvrant ses épaules ${ }^{49}$. La figure représentée sur la monnaie est habillée d'un long chiton serré en dessous des seins bien gonflés. La figure représentée sur cette monnaie n'est donc pas Apollon mais une femme.

Une monnaie de Colophon appartenant à la même série que notre monnaie et portant comme nom de magistrat $\Pi \mathrm{r} \Theta E O \Sigma$, a été datée de 190-30 av. J.-C. ${ }^{50}$. La figure représentée au revers a été considérée comme Apollon ${ }^{51}$. Pourtant la coiffure en chignon et les seins assez gonflés montrent qu'il s'agit d'une femme et pas d'Apollon. L'instrument

48) Doğan Gürbüzer 2012 : 184-185, nºs 21-28.

49) Dewailly 2001: 376, fig. KL.96.15. Léto coiffée du kredemnon a été souvent figurée sur les vases. Voir Berlin, Pergamonmuseum, inv. $\mathrm{n}^{\circ}$ F2641 ; Philadelphia (PA), University of Pennsylvania, no 5399 ; Munich, Antikensammlungen, $\mathrm{n}^{\circ} 1579$.

50) $S N G$ v. Aul., $\mathrm{n}^{\circ} 2017$.

51) $S N G$ v. Aul., n $2017 .$. 


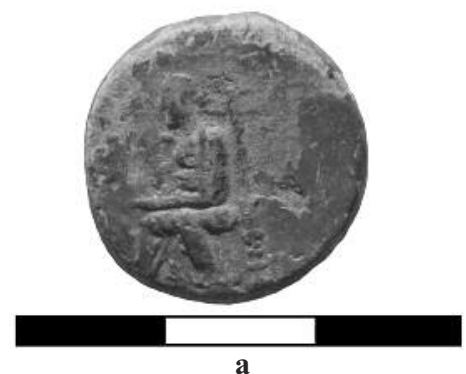

Fig. 4 : Monnaie provenant du sondage $R 13$ n$^{0} 2009.41$ (cliché N. Şahin).

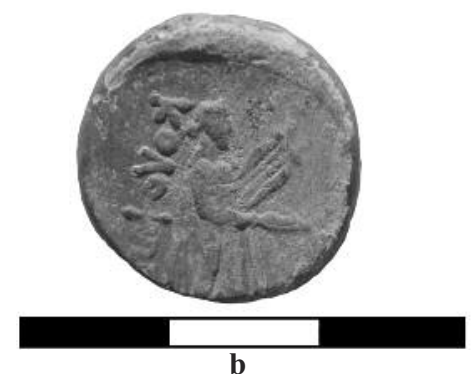

qu'elle tient n'est pas la lyre, mais la cithare et l'objet figuré dans sa main droite n'est pas un plectre mais une patère. La figure représentée au revers a été toujours identifiée comme Apollon sur quatre autres monnaies appartenant à cette même série ${ }^{52}$. Cependant cette figure porte toujours un long kredemnon et les seins bien visibles plus particulièrement sur les monnaies $\mathrm{n}^{\text {os }}$ 2918-2919 montrent que ce personnage n'est pas Apollon mais une femme. Il faut toutefois noter que la cithare et la patère ont été correctement identifiées sur ces monnaies.

$\mathrm{Ph}$. Kinns étudiant les monnaies de Colophonsur-Mer a recensé cent deux monnaies pour la série où apparaissent les deux magistrats $\mathrm{A} \mathrm{O} \Lambda \Lambda \mathrm{A} \Sigma$ et $\Pi$ П $\Theta E O \Sigma^{53}$. Le personnage représenté au revers a été aussi considéré comme Apollon par $\mathrm{Ph}$. Kinns ${ }^{54}$.

Sur une monnaie inédite provenant de la nécropole de Colophon-sur-Mer (Notion) dans le champ gauche le nom du magistrat mentionné est $\mathrm{A} \Pi \mathrm{O} \Lambda \Lambda \mathrm{A} \Sigma^{55}$

Après $\mathrm{Ph}$. Kinns, Z. Çizmeli Öğün a étudié deux monnaies appartenant à la même série, trouvées lors des campagnes de 1988-1997 à Claros ${ }^{56}$. Elle aussi a identifié comme Apollon la figure représentée au revers. Pourtant celle-ci toujours coiffée d'un chignon et d'un kredemnon est une femme. Elle a noté que le seul magistrat mentionné sur les monnaies était $\mathrm{A} \Pi \mathrm{O} \Lambda \mathrm{A} \Sigma^{57}$.

Dix monnaies trouvées lors des campagnes 20012007 ont été étudiées par İ. Önol dans son mémoire de DEA 2009 (inédit). Il a pu relever le nom du ma- 


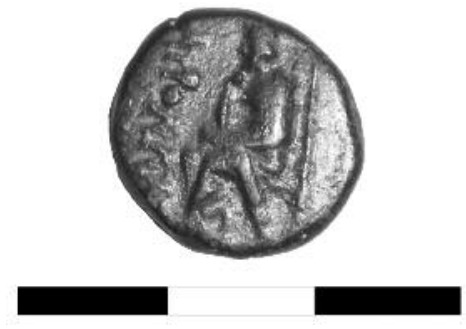

a

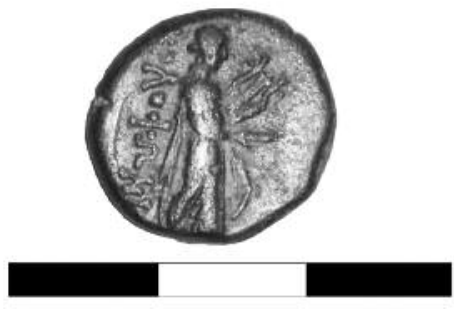

b

Fig. 5 : Monnaie, acquisition. Musée de Selçuk en exposition (Photos prises par O. Zunal avec la pemission du Musée de Selçuk).

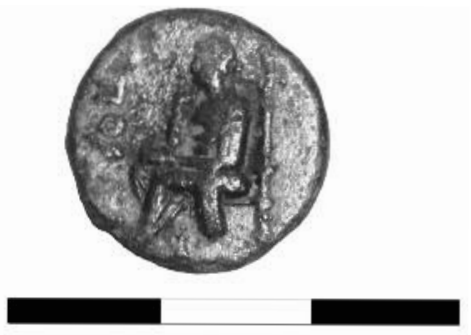

a

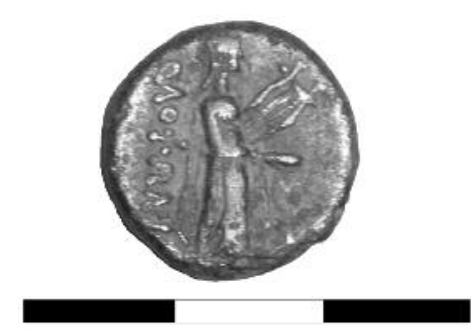

b

Fig. 6 : Monnaie, acquisition. Musée de Selçuk, en exposition (Photos prises par O. Zunal avec la pemission du Musée de Selçuk).

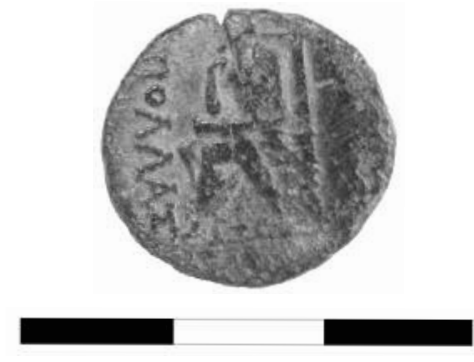

a

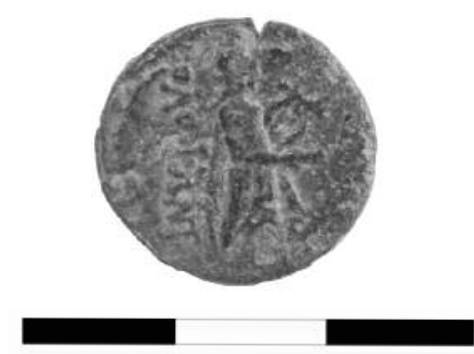

b

Fig. 7 : Monnaie, acquisition. Musée de Selçuk, au dépôt (Photos prises par O. Zunal avec la pemission du Musée de Selçuk). 


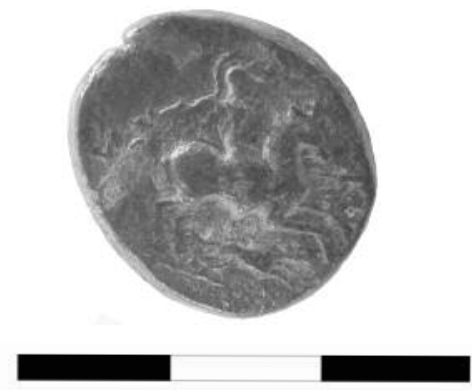

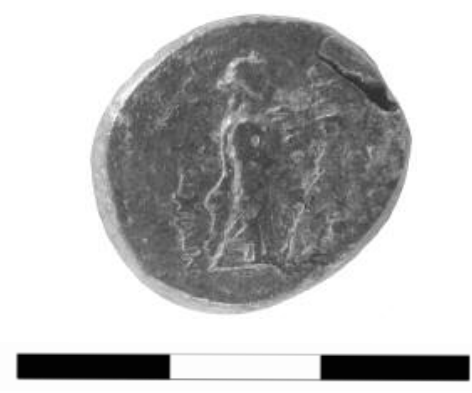

b

Fig. 8 : Monnaie provenant du sondage L15. Mus Inv. n $^{0} 38026$ (cliché N. Şahin).

L'iconographie d'une autre série de monnaies en bronze frappées par Colophon-sur-Mer mérite d'être étudiée encore plus attentivement. Elle présente comme type de droit un cavalier vêtu d'une chlamyde flottante, armé d'une lance dans la main droite ; le cheval est au galop ${ }^{61}$ à droite tandis qu'un chien courant à droite est représenté entre les jambes du cheval. Comme type de revers sur cette série se trouve une figure représentée debout à droite, une patère dans la main droite, une cithare dans la gauche, un trépied devant la figure dans le champ, à gauche l'ethnique, à droite le nom de magistrat $\mathrm{MHTP}[\mathrm{O} \Delta \Omega \mathrm{PO} \Sigma]$ (Fig. 8a-b) ${ }^{62}$. Nous voudrions attirer tout d'abord l'attention sur le chien figuré en dessous du cheval considéré comme un loup par J.G. Milne ${ }^{63}$. Par la suite Ph. Kinns a constaté qu'il s'agissait d'un chien et non d'un loup ${ }^{64}$. Quant à la figure représentée comme type de revers, elle a été considérée comme Apollon à la lyre par J.G. Milne ${ }^{65}$. Il soulignait que le dieu Apollon figuré au revers se trouvait placé dans un second rang. L'identification d'Apollon proposée encore par J.G. Milne a été acceptée par les numismates sans étude iconographique ${ }^{66}$. K. Konuk a publié une monnaie similaire et il a présenté dans son catalogue le personnage au revers comme un Apollon ${ }^{67}$.
Z. Çizmeli Öğün a étudié quatre monnaies appartenant à la même série trouvées lors des campagnes de 1990 et 1996 à Claros $^{68}$. Elle présente dans son catalogue le personnage au revers comme un Apol$l{ }^{69}$, alors que la figure coiffée toujours d'un chignon et d'un kredemnon est aussi une femme sur ces monnaies ${ }^{70}$.

La monnaie en bronze bien conservée provenant de Claros trouvée en $2007^{71}$ (Fig. 8a-b) montre clairement que la figure représentée au revers avec les cheveux en chignon ${ }^{72}$, la stéphanè et le kredemnon est comparable aux personnages placés sur les monnaies de Colophon-sur-Mer dites "Homèreion" et que l'on vient d'étudier ci-dessus (Fig. 1a-b ). Il faut souligner que ce sont des femmes qui sont toujours coiffées d'une stéphanè et d'un kredemnon. On aperçoit aussi que la figure a un sein bien gonflé donc nous sommes encore en présence d'une figure féminine et non pas d'Apollon d'une part et d'autre part elle tient une cithare dans la main gauche et non pas une lyre. Quatre monnaies provenant toujours de Claros trouvées en 1990 et 1996 montrent que ces figures sont similaires à la figurine représentée au revers sur la monnaie de $2007^{73}$. Un autre spécimen identique conservé dans la collection de Muharrem Kayhan, a été considéré comme Apollon

61) A propos du cheval sur les monnaies, voir Rousset 2014 : 57-60.

62) Milne 1941 : période VII B, groupe A, nos 169,172 ; Kinns 1980 : Æ $22, n^{\text {os }} 171-172$.

63) Milne $1941: 20$.

64) Kinns $1980: 334, n^{\circ} 172$.

65) Milne $1941: 76$.

66) Kinns 1980 : 584. SNG Tübingen, $n^{\circ} 2914$; SNG Kayhan, ${ }^{\circ} 387$; Çizmeli Öğün 2007 : 220 ; Önol 2009 : $53-54$; Delrieux $2014: 145, n^{\text {os }} 137-143$.

67) Konuk 2002 : Pl. 15, no 387.

68) Çizmeli Ögün 2007 : 220, Pl. LVII, nºs 37-39 ; Kinns 171-172 ; AE 22=Milne Période VII, Groupe B 169, 172.

69) Çizmeli Öğün $2007: 221$.

70) Çizmeli Öğ̈̈n $2007:$ Pl. LVII, $n^{\text {os }} 37-39$.

71) Önol $2009: \mathrm{n}^{\circ} 107$; Delrieux $2014: 145,179, \mathrm{n}^{\circ} 138$.

72) Léto a été souvent représentée avec les cheveux en chignon. Voir $A R V^{2}, n^{\text {os }} 617.1$ et 1132.180.

73) Çizmeli Öğün 2007 : fig. LVII, $n^{\text {os }} 37-39$. 


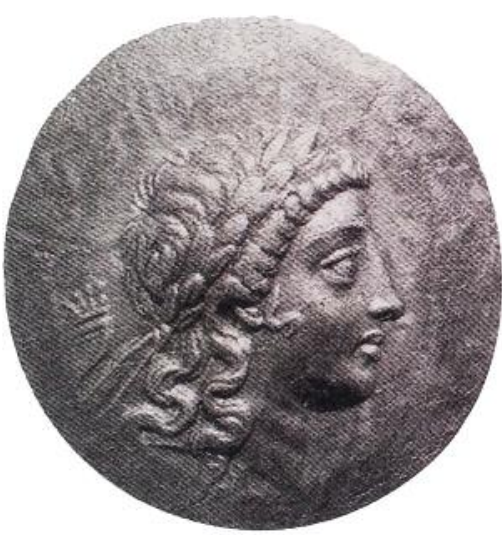

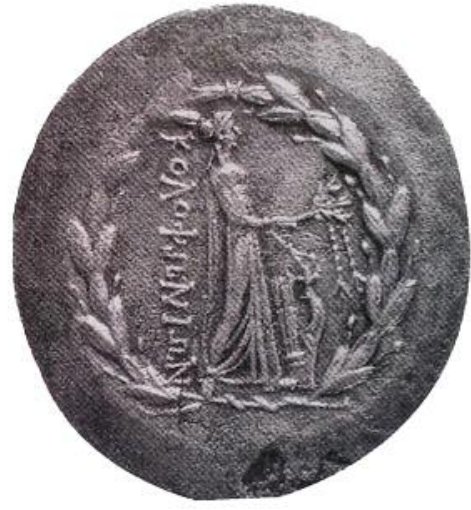

b

Fig. 9 : Monnaie de la collection de Muharrem Kayhan (Konuk 2002 : P1. 15, nº 386).

par K. Konuk ${ }^{74}$. Deux magistrats ont été mentionnés sur cette série : MHTPO $\Delta \Omega P O \Sigma$ et ЕПIГONO $\Sigma$.

Venons maintenant sur une autre série des monnaies en argent frappées par Colophon-sur-Mer. Il faut noter que les fouilles menées entre 2001 et 2015 ne nous ont pas fourni de monnaies de cette série. Cependant la figure représentée comme type de revers sur cette série est similaire à celles deux séries étudiées ci-dessus. C'est la raison pour laquelle nous avons décidé de faire une étude iconographique aussi pour ces monnaies. La tête d'Apollon au laurier tourné à droite constitue le type de droit. Quant à la figure représentée comme type de revers, elle a été considérée comme un Apollon par J.G. Milne ${ }^{75}$. Le personnage debout à droite tient une branche de laurier ${ }^{76}$ dans chacune de ses mains ; une cithare est posée du côté gauche de la figure, l'ethnique est à droite dans une couronne de laurier. La cithare a été identifiée comme une lyre par J.G. Milne ${ }^{77}$. Deux spécimens identiques à ceux de J.G. Milne sont donnés dans le catalogue de $\mathrm{Ph}$. Kinns ${ }^{78}$ et un exemplaire dans le catalogue de Konuk ${ }^{79}$. Les personnages présentés dans le catalogue de $\mathrm{Ph}$. Kinns et celui de Konuk ont toujours été identifiés comme des Apol- $\operatorname{lon}^{80}$. Pourtant l'exemplaire bien conservé provenant de la collection de Muharrem Kayhan montre clairement que la figure a été représentée de profil, avec ses cheveux en chignon ${ }^{81}$, coiffée toujours d'une stéphanè et d'un long kredemnon qui descend derrière son dos (Fig. 9a-b). Elle est habillée d'un chiton serré en dessous des seins bien gonflés ce qui conduit à penser que nous sommes encore en présence d'une femme et non pas d'Apollon. De plus, à notre connaissance, Apollon n'a été jamais représenté comme type de droit et de revers sur une même monnaie.

Abordons enfin l'iconographie de la dernière série de Colophon-sur-Mer. Il s'agit d'une monnaie d'Auguste en bronze provenant de Claros, trouvée en $2003^{82}$ (Fig. 10a-b). La tête laurée d'Auguste ${ }^{83}$ tournée à droite constitue le type de droit, avec $\Sigma \mathrm{E}$ $\mathrm{BA} \Sigma[\mathrm{TO} \Sigma]$ à gauche, une figure, avec les cheveux toujours en chignon, assise sur un trône, tenant une branche de laurier dans la main droite, une lyre ${ }^{84}$ dans la gauche comme type de revers, l'ethnique en deux rangs à gauche. Le premier exemplaire de cette série a été étudié par J.G. Milne ${ }^{85}$. La figure représentée au revers a été toujours considérée

74) Konuk $2002:$ P1. 15. 387.

75) Milne 1940 : période VII, groupe A, 164.

76) Voir Léto tenant une branche de laurier: $A R V^{2}, \mathrm{n}^{\text {os }} 617,611.32$ (peintre de Niobide) et 594.59.

77) Milne 1940 : période VII, groupe A, 164.

78) Kinns 1980 : période IV, AR VII, nº 162.

79) Konuk $2002: n^{\circ} 386$. Le numéro de l'exemplaire de Kinns a été donné comme 164 au lieu de 162 par Konuk.

80) Konuk 2002 : fig. $15,386$.

81) Léto a été souvent représentée avec les cheveux en chignon. Voir $A R V^{2}, \mathrm{n}^{\circ} 617.1$

82) Önol 2009 : 140, lev. VII, $\mathrm{n}^{\circ} 123$ = Delrieux $2014: 146, \mathrm{n}^{\circ} 162$.

83) Auguste s'est représenté comme Apollon. Cf. Graf 2005 : 426.

84) Delrieux (2014:146, $\left.n^{\circ} 162\right)$ a considéré cet instrument comme une cithare mais c'est une lyre.

85) Milne $1941: 82, \mathrm{n}^{\circ} 180$. 


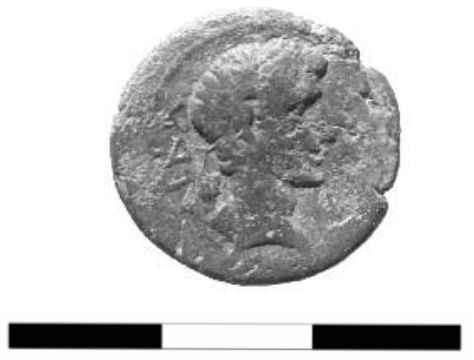

a

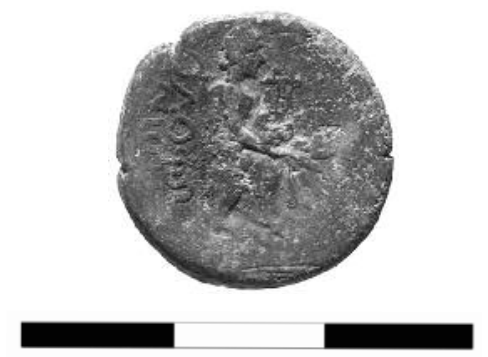

b

Fig. 10 : Monnaie provenant du sondage P14, $\mathbf{n}^{0} 8.1$ (cliché N. Şahin).

comme Apollon (assis) par J.G. Milne ${ }^{86}$ et cette identification a été acceptée par İ. Önol ${ }^{87}$ et F. Delrieux $^{88}$. Le personnage, avec les cheveux en chignon évoquant une déesse coiffée d'une stèphanè et avec le sein bien gonflé est là encore une femme et non pas Apollon.

Les monnaies autonomes de Colophon-sur-Mer étudiées ont montré qu'Apollon n'y a jamais été représenté comme type de revers bien qu'il soit fréquent sur les monnaies romaines (impériales) de Colophon-sur-Mer.

Il reste évidemment à intégrer à ce raisonnement la discordance iconographique relevée à propos de l'identification du personnage. Il est temps maintenant d'identifier la femme représentée sur ces quatre séries de monnaies autonomes de Colophon-surMer. Qui est cette figure féminine?

Le premier point de repère nous est fourni par deux fosses de dépôt trouvées au Sud du temple d'Apollon ${ }^{89}$. Parmi les trouvailles provenant des fosses se trouvent des statuettes féminines coiffées d'un polos, d'une stéphanè et d'un kredemnon ${ }^{90}$ qui tiennent une place importante dans l'iconographie féminine de Claros.

Un deuxième point de repère nous est fourni par un nouveau temple archaïque découvert dans le même secteur lors des campagnes menées entre 2012 et 2014.

A partir du milieu du $6^{\mathrm{e}}$ siècle commence une période de changements, un premier "âge d'or" du sanctuaire de Claros avec une transformation monumentale et l'instauration d'un nouveau culte d'Apollon Pythien venant de Delphes ${ }^{91}$. Roland Etienne $^{92}$ distingue trois moments dans l'histoire d'un lieu sacrée. L'un d'entre eux, c'est l'instauration d'un culte avec un prêtre et l'organisation d'un grand sanctuaire qui convient à Claros devenu alors l'un des grands sanctuaires apolliniens extra-urbains. Le plan d'ensemble du sanctuaire est l'objet d'une réfection globale : un temple et un grand autel rectangulaire dédiés à Apollon, un édifice placé au Nord du temple d'Apollon et un autel rectangulaire placé au Nord-Est du temple d'Apollon, consacrés à Artémis, un autre autel trouvé en 2009 au Sud de l'autel archaïque d'Apollon avec un mobilier qui ne permet pas déterminer la divinité à laquelle il était consacré ${ }^{93}$, un temple archaïque découvert au Sud du temple d'Apollon lors des dernières campagnes.

Revenons maintenant sur le nouveau temple archaïque trouvé au Sud du temple d'Apollon. A qui attribuer ce temple archaïque ? Parmi les trouvailles

86) Milne $1941: 82$

87) Önol $2009: 140$.

88) Delrieux $2014: 146, \mathrm{n}^{\circ} 162$.

89) Şahin $2014: 13$.

90) Şahin $2014: 13$, fig. 5-9.

91) Şahin et Debord $2011: 176$

92) Etienne et Prost $2008: 84$.

93) Şahin et Debord 2011 : 176, 199, fig. 19. Une base de korè no 1 (le bas du vêtement et les pieds conservés) provenant de Claros, découvert près des autels superposés d'Artémis en 1997. Le socle de la base d'origine est perdu mais korè était posée plus tard sur une dalle en calcaire. Cf. Pécasse 2004 : 21. Pécasse $(2004: 58)$ a daté cette korè dans le second tiers du $6^{\mathrm{e}}$ siècle comme deux dédicaces (korè et kouros) par Timonax. Selon elle, la korè $\mathrm{n}^{\circ} 1$ retenait son vêtement de la main droite. Cf. Pécasse $2004: 21$ korè $\mathrm{n}^{\circ}$ 1, Pl. 1.1. Cette constatation de Pécasse attire notre attention sur Léto représentée très souvent tenant son vêtement de la main droite ou de l'autre main sur les vases à figures noires et à figures rouges. Voir $A B V, \mathrm{n}^{\text {os }} 316.2,374.192,398.5 ;$ ARV $^{2}, \mathrm{n}^{\circ} 168 ; C V A$ Brussel, Musées Royaux d'Art et d'Histoire 2, III.H.E.9, Pl. (058) 18.5A.5B ; CVA New York Metropolitan Museum of Art, 4, 35, Pl. (762) 34.14 ; LIMC II, Pl. 233- 34, 634B, 641 A, B. Peut-on suggérer que Timonax avait dédié aussi une korè (korè $\mathrm{n}^{\circ} 1$ ) à Léto (évoquant ses images sur les vases) ? Il avait offert trois statues : deux korai, un kouros pour la triade. Serait-il possible que le socle de la base d'origine perdu fut placé à côté du nouvel autel archaïque dédié à Léto ? Car le deuxième socle en calcaire de la base de la statuette trouvé près de l'autel d'Artémis met en évidence que la place originale de statuette n'était pas là. 


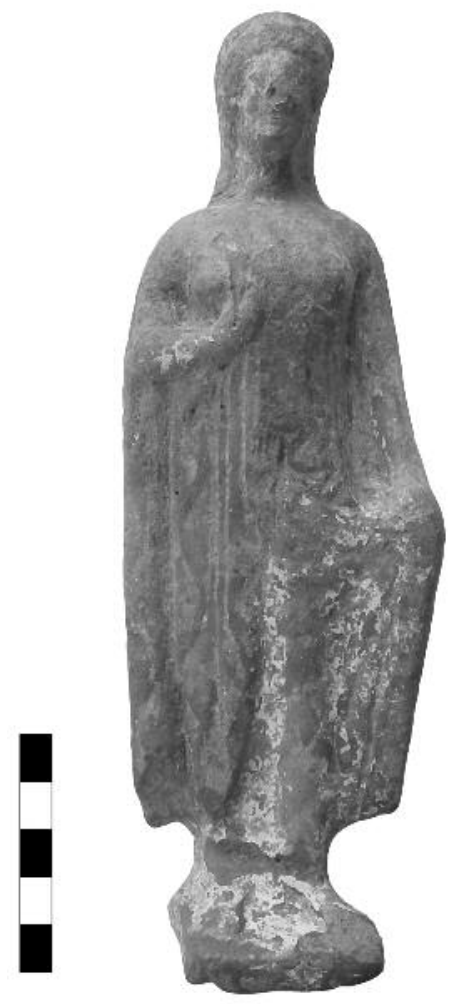

Fig. 11 : Statuette féminine découverte dans le temple de Léto, $\mathbf{n}^{0} 16.1$ (cliché N. Şahin).

provenant du naos du nouveau temple on compte deux statuettes féminines (Fig. 11) identiques aux trois figurines découvertes autour des autels d'Artémis (Fig. 12) ${ }^{94}$ et douze pendentifs (Fig. 13) appartenant à une parure féminine, eux aussi similaires aux quatre pendentifs toujours provenant du secteur d'Artémis (Fig. 14) ${ }^{95}$. M. Dewailly avait suggéré que Léto possédait un secteur cultuel au Sud du temple d'Apollon, en symétrie avec celui d'Artémis au Nord ${ }^{96}$. Le mobilier similaire provenant du secteur d'Artémis (au Nord du temple d'Apollon) et celui du BOA (au Sud du temple d'Apollon) confirme l'hypothèse de $M$. Dewailly par la présence du même type d'ex-voto offert aux deux divinités : à Léto et à sa fille Artémis. Nous sommes donc en présence du temple archaïque dédié à Léto découvert au Sud du temple d'Apollon. Ce temple, détruit (destruction des Perses) par un incendie, a fourni un matériel très abondant. Cependant une observation faite sur le temple mérite d'être signalée car elle

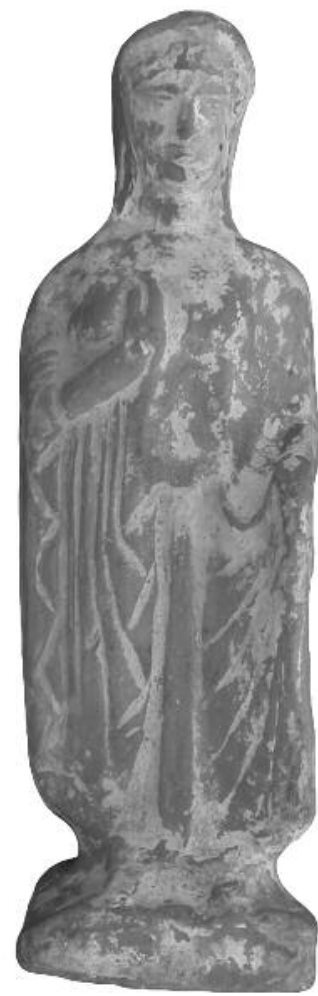

Fig. 12 : Statuette féminine provenant de l'autel d'Artémis (cliché N. Şahin).

atteste aussi deux phases de l'édifice. Il contenait plus de deux cents statuettes féminines et aussi trois cents têtes isolées coiffées de poloi et de stéphanai la plupart provenant du naos. Les figurines les plus anciennes datent de la fin du $6^{\mathrm{e}} \mathrm{s}$. av. J.-C. Les statuettes, bien conservées, relèvent de plusieurs types, produits en série. Coiffées de poloi, évoquant Léto coiffée du polos (Fig. 15), de la stéphanè (Fig. 16) et du kredemnon (Fig.17), elles sont débout, tenant une lyre (Fig. 15-16), une cithare dans la main gauche, un plectre souvent dans l'autre (Fig. 17). Elles représentent une déesse et plus particulièrement Léto. La présence d'un culte rendu à Léto dans le sanctuaire était assurée par la statue monumentale depuis les fouilles de Louis Robert. Cependant il a également été noté que cette statue étudiée par Jean Marcadé, portait un long châle (kredemnon $)^{97}$. Elle était voilée ${ }^{98}$ comme les figurınes provenant du temple du Léto. Marcadé a signalé que le châle (kredemnon) était relevé par le bras gauche ${ }^{99}$. Cette

94) Şahin $2014: 14$.

95) Şahin $2014:$ 14, 24, fig. 18-19.

96) Dewailly $2001: 367$.

97) Marcadé 1994 : 450-453.

98) Marcadé 1994 : 450.

99) Marcadé 1998 : 307. Léto a été représentée très souvent tenant son vêtement de la main droite ou gauche sur les vases. Cf. $A B V, \mathrm{n}^{\text {os }} 3254,301762$ (Metropolitan Museum : 67.44.), Altenburg, Staatliches Lindenau-Museum 22, CVA Brussels, $\mathrm{n}^{\circ}: 18.5 \mathrm{~A} .5 \mathrm{~B}$. , CVA London British Museum, $\mathrm{n}^{\circ} \mathrm{E} 256$. 


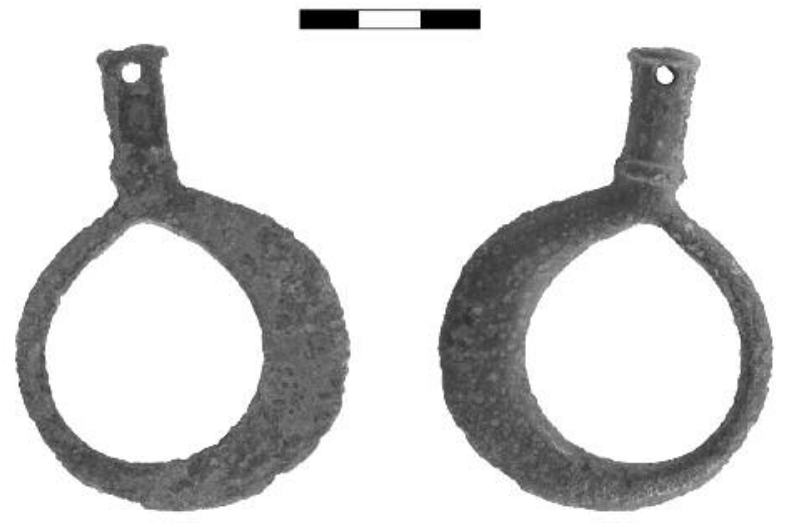

Fig. 13 : Pendentif provenant du secteur de BOA, $\mathbf{n}^{0} 19.25$ (cliché N. Şahin).

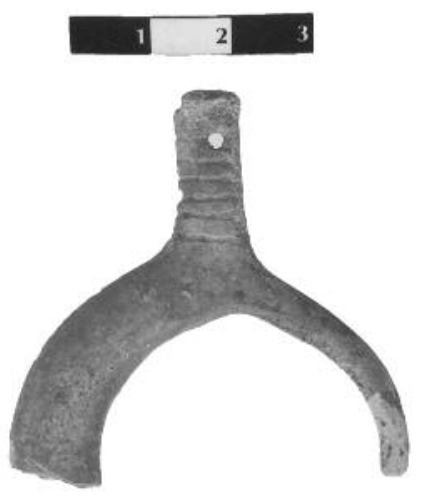

Fig. 14 : Pendentif provenant du secteur d'Artémis (cliché N. Şahin).
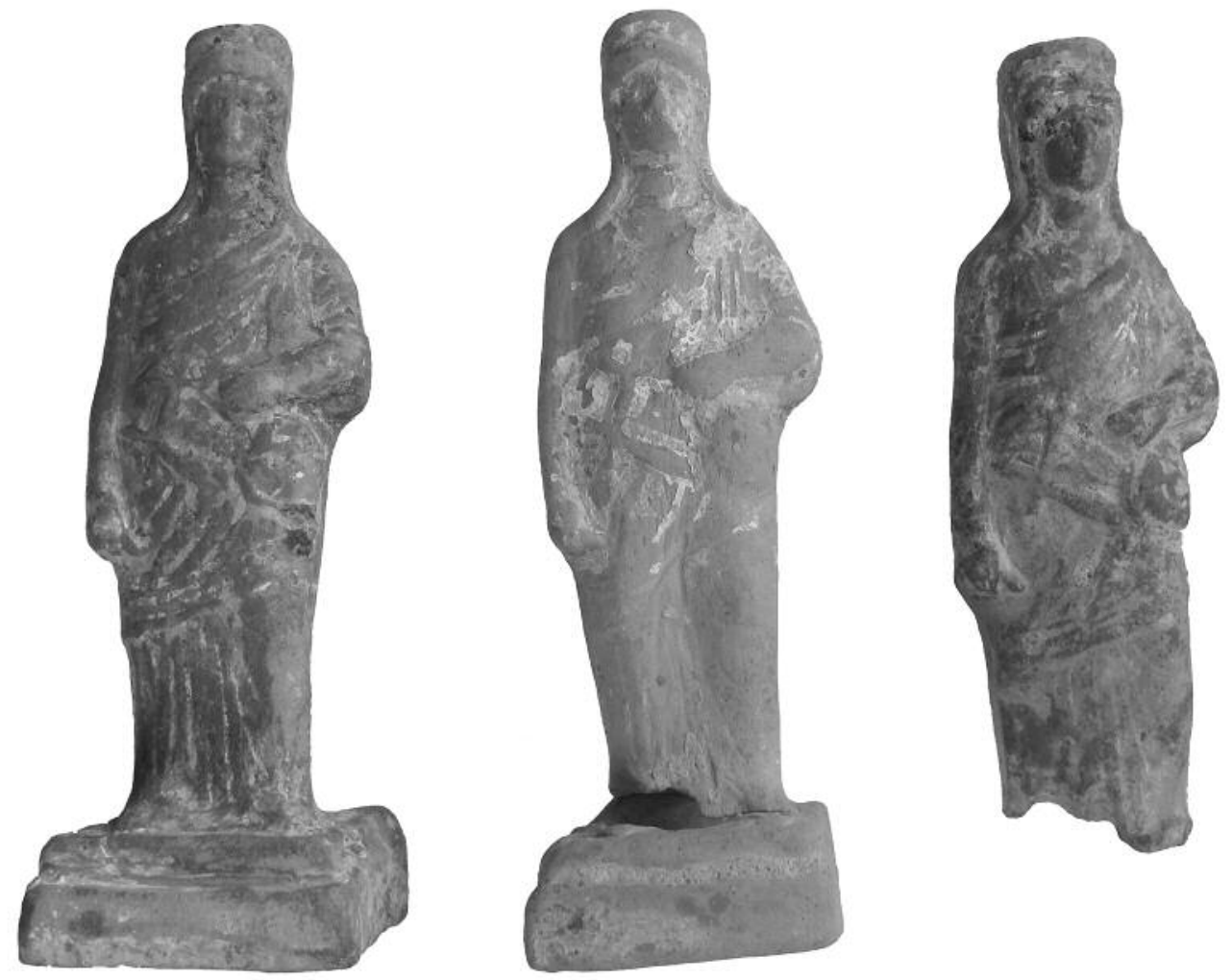

Fig. 15 : Déesses coiffées du polos (cliché N. Şahin).

statue monumentale a été datée de 170-160 av. J.-C. par Marcadé 100 au moment où les colophoniens-surMer (Notieis) ont établi des relations étroites avec les Attalides ${ }^{101}$.

La principale difficulté tient à l'activité de la déesse dans le sanctuaire. Sur ce point là un certain nombre de questions peuvent être posées : comment interpréter Léto à la lyre ou à la cithare ? Que signifie l'instrument de musique dans la main de la déesse ? Est-elle la déesse de la musique ou est-elle une divinité oraculaire de Claros ?

On peut encore signaler la présence de Léto parmi d'autres divinités sur un assez grand nombre de vases à figures noires et à figures rouges : elle y 

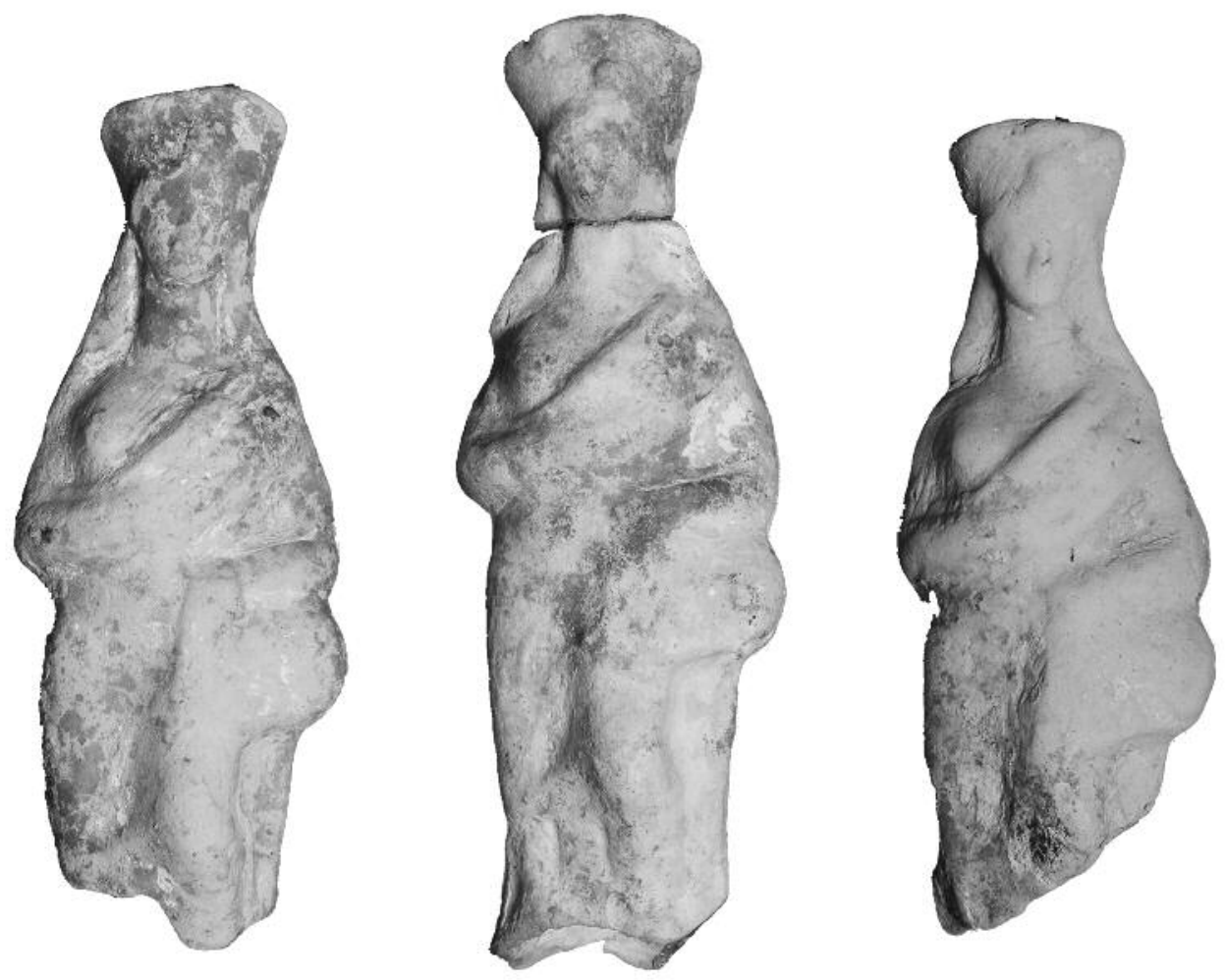

Fig. 16 : Déesses coiffées du stéphanè (cliché N. Şahin).

figure d'ordinaire à proximité d'Apollon ou d'Artémis et ne porte guère, comme signes distinctifs, que le kredemnon ${ }^{102}$ passé sur la tête en guise de voile, et un haut polos ${ }^{103}$ ou une stéphanè décorée de fleurons ${ }^{104}$; parfois aussi elle tient une branche de laurier ${ }^{105}$ ou une patère ${ }^{106}$ évoquant les figure féminines présentées sur certaines monnaies de Colophon-surMer étudiées ci-dessus.

A notre connaissance, aucune source littéraire n'atteste une activité musicale de Léto. Les sources iconographiques, cependant, illustrent cette activité : un vase provenant d'Etrurie, daté de 500-450 av. J.C., conservé au Musée de Gregoriano Etrusco Vaticano $^{107}$ porte une représentation d'Apollon sur son char partant pour le pays des Hyperboréens ; sa mère Léto avec les cheveux en chignon, coiffée d'une taenia, joue une cithare alors qu'Artémis tient les torches.

Selon Porphyre de Tyre, il y avait deux sortes d'inspiration: "Certains sont saisis en écoutant les sons du rythme de l'aulos ou de cymbale et du tympanon, qui ont été unis avec le dieu. Ils inspiraient comme corybantes. C'était l'inspiration par la méthode de divination de Sabazios et de la Déesse Mère. Certains buvaient la source sacrée pour saisir l'inspiration (enthousiasme) comme le prêtre du dieu de Claros de Colophon (Apollon)" 108 . Puisque Mantô (fondatrice de Claros) était la première devi-

102) $A R V^{2}, \mathrm{n}^{\text {os }} 7.3 ; 1155.8 \mathrm{~A}, 1053.32 \mathrm{~A}$; CVA MUNICH, Museum Antiker Kleinkunst 8, 57-58, beilage D5, Pl. (1821-1822,1824) 403.1, 404.1-2, 406.3 ; LIMC VI/1, P1. 133, 36, 38, P1. 136, 69. Léto est recouverte par un kredemnon sur une hydrie à figures rouges provenant de la collection du duc de Luynes, donnée à la Bibliothèque nationale, datée de 460-450. Voir BnF, Monnaies, Médailles et Antiques, Luynes.672. Une tête féminine provenant du monastère de Daphnis (sanctuaire d'Apollon Pythios) a été datée de la fin du $6^{\mathrm{e}}$ /début du $5^{\mathrm{e}}$ s. av. J.-C. La tête est recouverte par un kredemnon. C'est la raison pour laquelle Ioannis Mylonopoulos (2012 : 240) a proposé que cette tête pourrait avoir appartenu à une statue de Léto.

103) Toledo $(\mathrm{OH})$, Museum of Art : 1956.70 ; Brussels, Musées Royaux, R240;ABV, no 268.26 et $335.1 ;$ LIMC, 129. 3.

104) $A B V, \mathrm{n}^{\circ} 372.160 \mathrm{~A} ; A R V^{2}, \mathrm{n}^{\text {os }} 617.1 \mathrm{~A}, 1155.8 \mathrm{~A}, 1032.54,1053.32 \mathrm{~A} ; L I M C \mathrm{VI} / 1, \mathrm{Pl} .133, \mathrm{n}^{\text {os }} 36,38, \mathrm{Pl} .136, \mathrm{n}^{\circ} 69$.

105) Palermo, Mus. Arch. Regionale, $2187 ; A R V^{2}, \mathrm{n}^{\circ} 617.1$ (Léto avec les cheveux en chignon, coiffée d'une stéphanè tient une branche de laurier et un sceptre dans la main gauche), 611.32 et 594.59 .

106) New York (NY), Metropolitan Museum : $24.97 .96 ; A R V^{2}, \mathrm{n}^{\circ} 594.59$.

107) Beazley $1963: n^{\circ} 572.87$.

108) Porphyre de Tyre, Epistula ad Anabonem 2. 2 (14). 


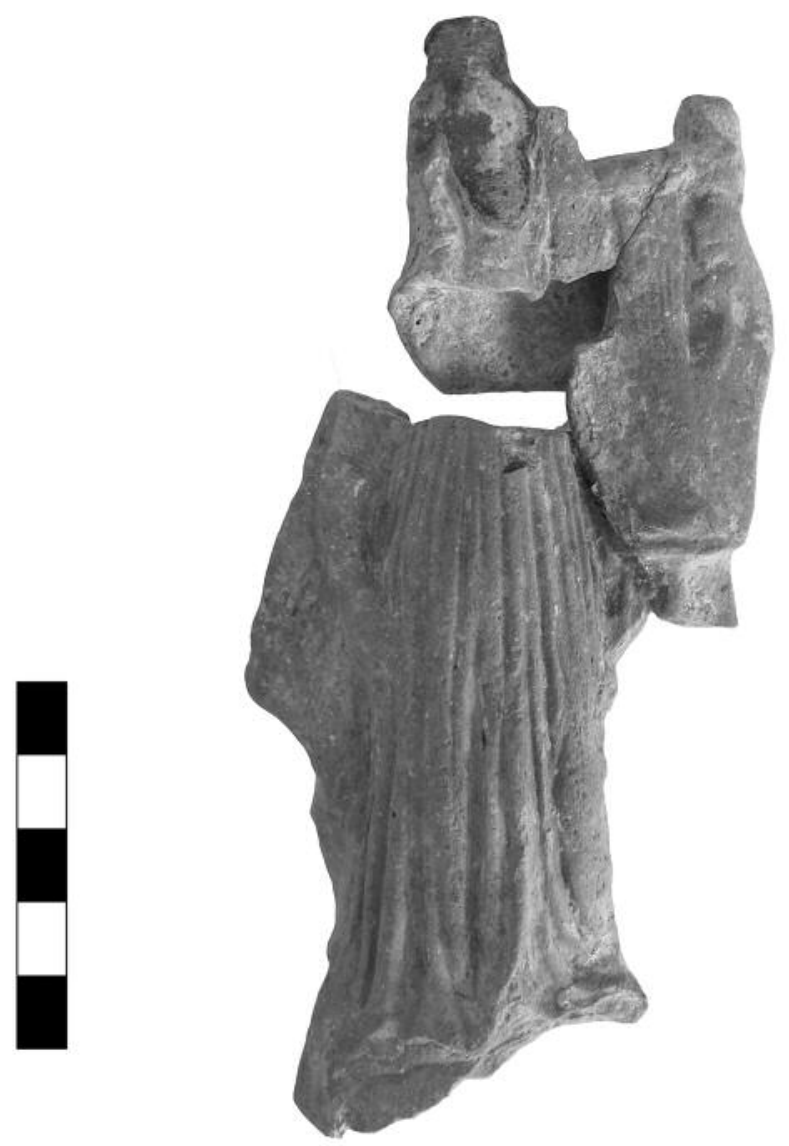

Fig. 17 : Déesse coiffée du kredemnon tenant une cithare (cliché N. Şahin).

neresse, elle buvait la source sacrée pour saisir l'inspiration. On sait que l'eau de la source sacrée était un élément principal dans la divination clarienne. Léto en tant que déesse anatolienne était une maîtresse des eaux et cela convient parfaitement à la divination clarienne. Cependant Jamblique ${ }^{109}$ dit qu'à Claros, "la présence du dieu prend comme instrument l'interprète qui ne s'appartient pas". D'après Philippe Monbrun ${ }^{110}$ "Quand Apollon inspire la Pythie, quand il joue de la lyre ou bien qu'il tire l'arc, Apollon est un 'Instrumentiste' qui s'empare d'un instrument saisi par l'enthousiame". M. Dewailly aussi suggérait que lorsqu'Apollon est représenté avec un instrument à corde, l'aspect musical était une métaphore de l'aspect oraculaire ${ }^{111}$. Peut-on aussi supposer que la présence des statuettes féminines à la lyre ou à la cithare représentant Léto montre la fonction oraculaire de la déesse ? Cette hypothèse ne serait pas étonnante parce que Léto avait un temple oraculaire à Bouto en Egypte. U. Pestalozza souligne que Bouto fut un centre important du culte de Léto, divinité oraculaire, déesse mère et nourrice comme Gaia à Delphes ${ }^{112}$. Il faut rappeler également que Mantô, première devineresse, avait rendu l'oracle de Niobé à Claros ${ }^{113}$. Selon Ovide ${ }^{114}$, cet oracle fut émis par Mantô, mais c'est Latoné elle-même qui, par la bouche de Mantô, (ore meo Latona iubet. Paretur et omnes) avait commandé aux Thébaines de célébrer une fête dédiée à elle et à ses enfants ${ }^{115}$. Nous sommes donc en présence d'une Léto oraculaire à Claros comme Gaia à Delphes. Mantô apparaît comme un instrument de communication entre les consultants qui interrogent et le dieu qui répond. $\mathrm{Ph}$. Monbrun considère Apollon comme instrumentiste donc "Quand Léto inspire la Mantô, quand elle joue de la lyre ou de la cithare, Léto aussi est un 'Instrumentiste' qui s'empare d'un instrument saisi par l'enthousiasme". C'est Léto qui était la déesse oraculaire avant Apollon (son fils) à Claros. Quand son fils est-il devenu le dieu oraculaire de Claros? Selon Conon, "Mopsos disait qu'il avait pris le centre oraculaire de Claros après la mort de sa mère" 116 . Il semble que c'était le temps où Mantô mourut et Mopsos devenu devin au moment où Léto donnait la main à son fils. Cependant on sait qu'Apollon était absent pendant trois mois. Est-il possible que pendant l'absence d'Apollon, l'oracle fonctionnait par l'intermédiaire de Léto à Claros?

\section{CONCLUSION}

Selon Milne, Apollon "le dieu principal" représenté au revers était placé au deuxième rang sur les monnaies de Colophon-sur-Mer. Pourtant les figurines à la lyre et à la cithare en terre cuite montrent très clairement que la figure à la cithare et à la lyre re-

109) Jamblique, De Mysteriis, III, 11.

110) Monbrun $2007: 273$.

111) Dewailly $2001: 373$.

112) Pestalozza $1942: 80$ sq.

113) Sahin $2008: 218-219$.

114) Ovide, Mét., VI. 160-165.

115) Un oracle similaire a été donné par Apollon aux Colophoniens de célébrer la fête dans le décret du koinon ionien provenant de Claros daté du $3^{\mathrm{e}}$ siècle av. J.-C. Voir Etienne et Prost 2008 : 84-85.

116) Conon, 6. 
présentée sur les monnaies de Colophon-sur-Mer comme type de revers n'était jamais Apollon mais Léto oraculaire. Léto représentée sur ces monnaies est l'un des types par excellence des quatre groupes de monnaies de Colophon-sur-Mer.

Le changement de personnages et de types est en relation avec l'histoire politique de Colophon l'Ancienne. Le nom de Notion a varié suivant les époques ${ }^{117}$. Pendant le $6^{\mathrm{e}}$ siècle, elle s'appela Notion ${ }^{118}$ et Colophon-sur-Mer. La ville devint enfin Colophon tout court et le nom de Notion sortit d'usage ${ }^{119}$. $\mathrm{D}$ 'après $\mathrm{Ph}$. Gauthier, à la fin du $4^{\mathrm{e}}$ siècle et pendant une bonne partie du $3^{\mathrm{e}}$ siècle, Colophon fut une cité à deux têtes ${ }^{120}$. Ph. Gauthier suppose qu'entre les années 250-230, la convention de sympolitie a été rendue caduque ${ }^{121}$. Cependant les dernières monnaies frappées par Colophon l'Ancienne ont été datées de 320-294. Après la déportation des habitants de Colophon l'Ancienne à Arsinoeia (Ephèse) et la destruction de la ville en 294 av. J.-C. par Lysimaque, l'atelier de Colophon l'Ancienne ne frappait plus de monnaies alors que Colophon-sur-Mer commence à en frapper. Le nouvel atelier décide du choix des nouveaux types, mais conserve l'ancien ethnique car Colophon-sur-Mer s'appela longtemps comme Colophon. Apollon n'a jamais été utilisé comme type caractéristique de revers des monnaies autonomes de Colophon-sur-Mer, mais il a été représenté sur les monnaies impériales de Colophon-sur-Mer.

La présence d'Homère représenté comme type de droit montre qu'il est originaire de Colophon. Homère patronnait aussi en quelque sorte la formation de la jeunesse à Colophon-sur-Mer ${ }^{122}$. La présence du gymnase dit "Homèreion" à Colophon-sur-Mer le prouve et l'on sait que les gymnases jouaient un rôle important dans l'idéologie du royaume attalide comme l'atteste notamment le décret en l'honneur d'Athénaios. L'atelier ou bien deux magistrats ( $\mathrm{A} \Pi \mathrm{O} \Lambda \Lambda \mathrm{A} \Sigma$ et $\Pi \mathrm{r} \Theta \mathrm{EO} \Sigma$ ) ont pu choisir Homère, patron du gymnase, comme nouveau type pour montrer leurs engagements envers la monarchie attalide.

Les trente-deux monnaies (groupe portant une représentation d'Homère) provenant de la strate de préparation du sol foulé lors des hécatombes montrent très clairement que leur datation donnée par $\mathrm{Ph}$. Kinns pose un problème chronologique. Les monnaies à l'effigie semblent avoir été frappées dans le deuxième quart du $2^{\mathrm{e}}$ siècle av. J.-C. au moment où sa statue a été exécutée et où le décret d'Athénaios fut voté.

N.Ş.

117) Piérart $1984: 170-171$.

118) Gauthier 2003: 81.

119) Charneux 1966: 195, 197.

120) Gauthier $2003: 78$

121) Gauthier $2003: 87$. Piérart $1984: 170-171$.

122) Gauthier $2006: 492$. 


\section{BIBLIOGRAPHIE}

Amandry, M., 1992 : "Le trésor monétaire du sondage 2a", in La Genière, J. (éd.), Cahiers de Claros 1, Paris : 91-99.

ABV : Beazley, J.D., Attic Black-Figure Vase-Painters, Oxford, 1956.

$A R V^{2}$ : Beazley, J.D., Attic Red-Figure Vase-Painters, $2^{\text {nd }}$ ed., Oxford, 1963.

BMC, Ionia : Head, B.V. , A Catalogue of Greek coins in the British Museum, Ionia, Londres, 1892 (rééd., Bologne, 1964).

CVA Munich: Corpus Vasorum Antiquorum: Munich, Museum Antiker Kleinkunst.

CVA Brussel : Corpus Vasorum Antiquorum: Brussel, Musées Royaux d'Art et d'Histoire 2.

CVA New York: Corpus Vasorum Antiquorum: New York Metropolitan Museum of Art 4.

Charneux, P., 1966: "Liste argienne de théarodoques", BCH 90/1 : 156-239.

Çizmeli Öğün, Z., 2007 : "Les monnaies découvertes à Claros, sanctuaire d'Apollon en Ionie", $R N$ 163 : 213233.

- 2011 : “Les monnaies découvertes à Claros, sanctuaire d'Apollon en Ionie, 2. Commentaire historique et numismatique", $R N 167: 321-338$.

- 2012 : "Klaros Kutsal Alanı Kazılarında Ele Geçen Sikkeler (1988-1997)", in Yiğit, T., Kaya, M.A. et Sina, A. (éds.), Ömer Çapar'a Armağan, Ankara : 57-74.

Debord, P., 2013 : "Le pays de Colophon (Colophon, Claros, Notion) et les Séleucides", REA 115 : 5-27.

Delattre, L., La Genière, J. et Şahin, N., 2003 : "Le Sondage 1 B", in La Genière, J. et Jolivet, V. (éds.), Cahiers de Claros II. L'Aire des sacrifices, Paris : 1529.

Delrieux, F., 2014: “ " Les monnaies de fouilles trouvées à Claros en 2001-2011. Essai de circulation monétaire dans un sanctuaire oraculaire ", in Moretti, J.C. et Rabatel, L. (éds.), Le Sanctuaire de Claros et son oracle. Colloque International Lyon, Maison de l'Orient et de la Méditerranée Amphithéâtre Benveniste 13-14 Janvier 2012, Lyon : 133-188.

Demangel, R. et Laumonier, A., 1923 : "Fouilles de Notion", BCH 47 : 353-386.

Dewailly, M., 2001 : "Le sanctuaire d'Apollon à Claros : place et fonction des dieux d'après leurs images", MEFRA 113 : 366-382.

Doğan Gürbüzer, E., 2012 : Klaros Kazılarında Bulunmuş Olan Pişmiş Toprak Figürinler ve Kültler Açısından Değerlendirilmeleri, İzmir (thèse de doctorat inédit).

Etienne, R. et Prost, F., 2008 : “Claros, les modèles delphique au pays des Létoïdes", in Şahin, N., Aytaçlar, M.N., Baştürk, M.B., Dedeoğlu, F. et Erdoğan, A. (éds.), 1. Uluslararası Antik Dönemde Kehanet ve Apollon'un Anadolu Kültleri Sempozyumu. İmir 17-20 Ağustos 2005, [Arkeoloji Dergisi 2] : 75-88.

Gauthier, Ph., 1996 : "Bienfaiteurs du gymnase au Létoon de Xanthos", REG 109 : 1-34.
- 2003 : “Le décret de Colophon l'Ancienne en l'honneur du Théssalien Asandros et la sympolitie entre les deux Colophon", JSav : 61-100.

- 2006 : "Les décrets de Colophon-sur-Mer en l'honneur des Attalides

Athénaios et Philétairos ", REG 119 : 473-503.

Graf, F., 2005 : “Apollo”, Thomson Gale Encyclopedia of Religion ( $2^{\text {nd }}$ ed.) vol. 1, 424-426.

Habicht, C., 1969 : Inschriften von Pergamon VIII / 3.

Head, B.V., 1911 : Historia Numorum, Oxford.

Holleaux, M., 1906 : "Note sur une inscription de Colophon Nova", BCH 30 : 349-358.

Kinns, P., 1980 : Studies in the Coinage of Ionia: Erythrae, Teos, Lebedos, Colophon, c. 400-30 BC, (Ph. D. Diss.), Cambridge.

Knapp, R.C. et Isaac, J.D. Mac, 2005 :

Excavations at Nemea III. The Coins, California, University of California.

Konuk, K., 2002 : Sylloge Nummorum Graecorum, Turkey I. The Muharrem Kayhan Collection, Bordeaux. Kraemer, B., 2010 : "The Meandering Identity of a Fayum Canal: The Henet of Moeris /

Dioryx Kleonos / Bahr Wardan / Abdul Wahbi", Proceedings of the Twenty-Fifth International Congress of Papyrology, Ann Arbor 2007. [American Studies in Papyrology] : 365-376.

La Genière , J., 1996 : "Sanctuaire d'Apollon à Claros, 1995", CRAI : 261-272.

- 1998 : "Claros. Bilan provisioire de dix campagnes de fouilles", REA 100/1-2: 235-268.

-2003 : "Les états successifs de l'aire des sacrifices d'après la stratigraphie", in La Genière, J. et Jolivet, V. (éds.), Cahiers de Claros II. L'Aire des sacrifices, Paris : 181-209.

LIMC : Lexicon Iconographicum Mythologiae Classicae.

Malay, H., 1992 : Hellenistik Devirde Pergamon ve Aristonikos Ayaklanması, İzmir.

Marcadé, J., 1994 : "Rapport préliminaire sur le groupe cultuel du temple d'Apollon à Claros", REA 96 : 447-463.

- 1998 : "Nouvelles observations sur le groupe cultuel du temple d'Apollon à Claros", REA $100: 299-323$.

Milne, J.G., 1941 : Kolophon and its Coinage: Study, ANS Numismatic Notes and Monographs 96, New York.

Monbrun, P., 2007 : Les voix d'Apollon. L'Arc, la lyre et les oracles, Rennes.

Mylonopoulos, I., 2012 : “Chronique archéologique de la religion grecque", Kernos 25 : 233-295.

Önol, İ., 2009 : 2001-2007 Yilları Arasinda Klaros Kazılarında Ele Geçen Sikkeler (mémoire de DEA inédit), İzmir.

Pécasse, M., 2004 : “L'étude des sculptures”, Les sculptures archaïques de Claros, Monuments et Mémoires de la Fondation Eugène Piot 83 : 21-59. 
Pestalozza, U., 1942 : Pagine di religione mediterranea I, Milano.

Picard, C. et Macridy, T., 1915 : "Fouilles du Hiéron d'Apollon Clarios à Colophon. Première campagne", BCH 39 : 33-52.

Piérart, M., 1984 : "Deux notes sur la politique d'Athènes en mer Egée (428-425)", BCH 108/1 : 161176. 1970.

Robert, L., 1970 : Etudes Anatoliennes, Amsterdam,

Robert, L. et Robert, J., 1989 : Claros I. Décrets hellénistiques, Paris. 173-188.

Robert, M.R., 1999 : "Le poète de Claros", CRAI :

Rousset, D., 2014 : “La stèle des Géléontes au sanctuaire de Claros. La souscription et les acquisitions immobilières d'une subdivision civique de Colophon", Journal des Savants (Janvier-Juin) : 3-98.

SNG Cop. 1942-1979 : Sylloge Nummorum Graecorum-Denmark: The Royal Collection of Coins and Medals of the Danish National Museum, 43 facs., Copenhague.

SNG Tübingen 1989 : Sylloge Nummorum Graecorum-Deutschland: Münzsammlung der Universität Tübingen, 4. Heft : Mysien-Ionien, München.

SNG v. Aul., Ionien 1960 : Sylloge Nummorum Graecorum - Deutschland: Sammlung von Aulock, Ionien, 6. Heft, Berlin.

SNG Lewis 1972 : Sylloge Nummorum Graecorum, The Lewis Collection in Corpus Christi College, Cambridge, Part I, The Greek and Hellenistic Coins, Londres.
Şahin, N., 2003 : “Le sondage 1.H”, in La Genière, J. et Jolivet, V. (éds.), Cahiers de Claros II. L'Aire des sacrifices, Paris : 93-97.

- 2008 : "A la lumière découvertes récentes : relations de Claros avec Notion et Oracles de Manto", in Şahin, N., Aytaçlar, M.N., Baştürk, M.B., Dedeoğlu, F. et Erdoğan, A (éds.), 1. Uluslararası Antik Dönemde Kehanet ve Apollon'un Anadolu Kültleri Sempozyumu. İmir 17-20 Ağustos 2005, [Arkeoloji Dergisi 2] : 213226.

- 2014 : "Claros. Les Campagnes de 2010 et 2011 nouveaux apports" in Moretti, J.-C. et Rabatel, L. (éds.), Le Sanctuaire de Claros et son oracle. Colloque International Lyon, Maison de l'Orient et de la Méditerranée Amphithéâtre Benveniste 13-14 Janvier 2012, Lyon : 1331.

Jamblique, De Mysteriis (Aegyptiorum Chaldaeorum Assyriorum).

Conon, Narratium.

Ovide, Métamorposes.

Polybe

Porphyre de Tyre, Epistula ad Anabonem.

Strabon Geographica.

Socrate, Phèdre.

Tite Live, XXXVIII, 39-41.

Xénophon, Le Banquet. 


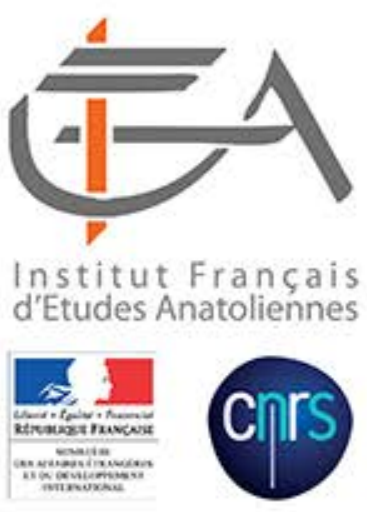

Eurômos : le temple de Zeus vu de l'Est (cliché Sönmez Alemdar).

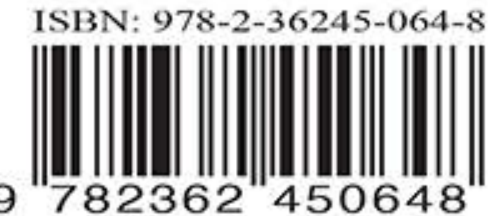

